\title{
Assessment of Metabolic Dysfunction in Sepsis in a Retrospective Single-Centre Cohort
}

\author{
Julien Goutay $\left(D,{ }^{1}\right.$ Juliette Perche, ${ }^{2}$ Aurelia Toussaint, ${ }^{1}$ Elodie Drumez, ${ }^{3}$ \\ Michael Howsam (iD, ${ }^{4}$ Claire Bourel, ${ }^{1}$ Benoit Brassart, ${ }^{1}$ Alexandre Pierre, ${ }^{1}$ Morgan Caplan, ${ }^{5}$ \\ Arthur Durand, ${ }^{6}$ Marion Houard, ${ }^{6}$ Saad Nseir, ${ }^{5}$ Raphael Favory, ${ }^{1}$ and Sébastien Preau ${ }^{1}$ \\ ${ }^{1}$ Division of Intensive Care, University Lille, CHU Lille, Inserm, Institut Pasteur de Lille, U1167, Lille 59000, France \\ ${ }^{2}$ Division of Intensive Care, CH Roubaix, Roubaix 59170, France \\ ${ }^{3}$ University Lille, CHU Lille, ULR 2694-METRICS: Évaluation des Technologies de Santé et des Pratiques Médicales, \\ Lille 59000, France \\ ${ }^{4}$ University Lille, Inserm, Institut Pasteur de Lille, U1167, Lille 59000, France \\ ${ }^{5}$ Division of Intensive Care, University Lille, CHU Lille, Lille 59000, France \\ ${ }^{6}$ Division of Intensive Care, CHU Lille, Lille 59000, France
}

Correspondence should be addressed to Julien Goutay; julien.goutay@gmail.com

Received 19 April 2021; Revised 1 August 2021; Accepted 29 November 2021; Published 20 December 2021

Academic Editor: Samuel A. Tisherman

Copyright ( $\odot 2021$ Julien Goutay et al. This is an open access article distributed under the Creative Commons Attribution License, which permits unrestricted use, distribution, and reproduction in any medium, provided the original work is properly cited.

Objective. Our primary aim was to assess selected metabolic dysfunction parameters, both independently and as a complement to the SOFA score, as predictors of short-term mortality in patients with infection admitted to the intensive care unit (ICU). Methods. We retrospectively enrolled all consecutive adult patients admitted to the eight ICUs of Lille University Hospital, between January 2015 and September 2016, with suspected or confirmed infection. We selected seven routinely measured biological and clinical parameters of metabolic dysfunction (maximal arterial lactatemia, minimal and maximal temperature, minimal and maximal glycaemia, cholesterolemia, and triglyceridemia), in addition to age and the Charlson's comorbidity score. All parameters and SOFA scores were recorded within $24 \mathrm{~h}$ of admission. Results. We included 956 patients with infection, among which 295 (30.9\%) died within 90 days. Among the seven metabolic parameters investigated, only maximal lactatemia was associated with higher risk of 90-day hospital mortality in SOFA-adjusted analyses (SOFA-adjusted OR, 1.17; 95\%CI, 1.10 to 1.25; $p<0.001)$. Age and the Charlson's comorbidity score were also statistically associated with a poor prognosis in SOFA-adjusted analyses. We were thus able to develop a metabolic failure, age, and comorbidity assessment (MACA) score based on scales of lactatemia, age, and the Charlson's score, intended for use in combination with the SOFA score. Conclusions. The maximal lactatemia level within $24 \mathrm{~h}$ of ICU admission is the best predictor of short-term mortality among seven measures of metabolic dysfunction. Our combined "SOFA + MACA" score could facilitate early detection of patients likely to develop severe infections. Its accuracy requires further evaluation.

\section{Background}

Sepsis is a well-recognized, worldwide healthcare issue defined as life-threatening organ dysfunction resulting from a dysregulated host response to an infection [1-3]. Septic shock is the worst form of sepsis, associated with severe hemodynamic (i.e. median arterial pressure $<65 \mathrm{mmHg}$ requiring vasopressor administration) and metabolic dysfunction (i.e. lactatemia $\geq 2 \mathrm{mmol} / \mathrm{L}$ ) [4]. This condition is characterized by an abrupt and massive release of stress hormones, leading to an overwhelming production of energy substrates in the form of glucose, fatty acids, amino acids, and lactate [5]. The most severe cases tend to exhibit elevated levels of plasmatic glucose [6] and triglycerides[7], while elevated lactatemia [4, 8-12], hypothermia [13], hypoglycaemia $[14,15]$, and hypocholesterolemia [16-18] have all been associated with poor 
outcomes in critically ill patients. All these parameters are easy to measure at the bedside and may help in early detection of severe infections. However, despite its significance in the pathophysiology of both septic shock and sepsis, metabolic dysfunction only forms part of the diagnosis for the former $[19,20]$.

Sepsis-related organ dysfunction is characterized by an acute change of $\geq 2$ points in the total Sequential Organ Failure Assessment (SOFA) score following infection, and this score is already in widespread clinical use [3]. However, the utility of commonly measured indicators of metabolic dysfunction as a complement to the SOFA score's prediction of short-term mortality in infected patients has yet to be studied, despite it forming part of the diagnosis of septic shock.

\section{Methods}

The primary aim of this study was to examine measurements of metabolic dysfunction, age, and the Charlson's comorbidity score (metabolic failure, age, and comorbidity assessment-MACA) as predictors of short-term mortality in patients with infection and to compare their predictive power with that of the already established SOFA score.

The secondary objective was to assess the utility of a composite score which combined the best performing of the MACA measurements with the SOFA score. We compared the relative diagnostic and prognostic performance of this combined "SOFA + MACA" score with that of the SOFA score alone, with the aim of generating a standardized, more generalized integration of metabolic dysfunction in the clinical management of cases of infection.

2.1. Study Design. We retrospectively enrolled all consecutive adult patients admitted to the eight intensive care units (ICUs) of the Intensive Care Centre (92 beds) in the University Hospital of Lille, between January $1^{\text {st }} 2015$ and September $30^{\text {th }} 2016$, with suspected or confirmed infection. A suspected infection was identified through the combination of antibiotic use ( $\geq 72$ hours) and a clinician requesting body fluid culture to be performed [3]. A proven infection was defined as the invasion of normally sterile tissue, fluid, or a body cavity by pathogenic or potentially pathogenic microorganisms [21]. We considered only the first admission during the study period. We identified patients by interrogating our electronic prescription support software (IntellisPace Critical Care and Anesthesia ICCA ${ }^{\circledR}$ version H.02.01, Philips, France). This observational study was performed in strict compliance with the French reference methodology MR-004, established by the French institutional authority for personal data protection (CNIL-Commission Nationale de l'Informatique et des Libertés). In accordance with ethical standards, informed consent was not necessary for demographic, physiological, and hospital-outcome data analyses because this observational study did not modify existing diagnostic or therapeutic strategies. The CNIL (DEC19-259) and our institutional review board (HP20/39) approved the methodology.
2.2. Data Collection. Data were obtained from the electronic medical record system of the Intensive Care Centre, focusing specifically on demographic data, comorbidities, and the Charlson's score [22]. Measured physiological variables were used to calculate the SOFA score [23]. Mild-to-severe liver disease and renal insufficiency were defined respectively by a Child-Pugh score of $\geq 7$ [24] and a creatinine clearance $<60 \mathrm{ml} / \mathrm{min} / 1.73 \mathrm{~m}^{2}$ of body surface [25]. Sepsis and septic shock were defined as previously described [2-4]. Community-associated infections were infections becoming manifest and being diagnosed within 48 hours of ICU admission in patients with no previous encounter with healthcare [26]. Healthcare-associated infections were defined as an infection that occurred while receiving care in a hospital or other healthcare facility, and that was neither present nor incubating upon admission [27]. Immunocompromised status was defined by the use of corticoids ( $>10 \mathrm{mg} /$ day of prednisone or its equivalent), the administration of chemotherapy, the use of non-steroidal immunosuppressive agents following organ transplantation, or a diagnosis of autoimmune disease [28]. At hospital discharge, we collected the Rankin score [29] and the Glasgow Outcome Scale (GOS) [30].

We preselected seven biological and clinical parameters of metabolic dysfunction, all routinely measured in the participating ICUs, namely maximal arterial lactatemia, minimal and maximal temperature, minimal and maximal glycaemia, cholesterolemia, and triglyceridemia. All metabolic parameters and SOFA scores were recorded within 24 hours after ICU admission. All lactatemia measurements recorded in this study were performed on arterial blood samples using devices in the ICU (ABL 90 Flex, Radiometer TM [31]). Age and the Charlson's comorbidity score were integrated with these 7 measurements of metabolic dysfunction to generate a new score representing MACA.

2.3. Statistical Analysis. Quantitative variables were expressed as medians (interquartile range, IQR). Normality of distributions was assessed using histograms and the Shapiro-Wilk test. Categorical variables were expressed as numbers (percentages). The magnitude of differences between groups according to 90-day mortality status was assessed using absolute standardized differences (ASD), with an ASD $>20 \%$ being interpreted as a meaningful difference. Because the present study additionally aimed to assess the utility of metabolic dysfunction parameters as a complement to the SOFA score, we specifically focused our analyses on metabolic parameters for which daily measurements were readily available. To account for two important predictors of mortality, age and the Charlson's comorbidity score were also included as candidate predictors of interest into the "MACA" score. We compared the MACA score between survivors and non-survivors (in-hospital mortality within 90 days) using unadjusted and SOFA-adjusted logistic regression models. For each parameter, the assumption of log-linearity for identical SOFA scores was verified using the cubic splines function. For 


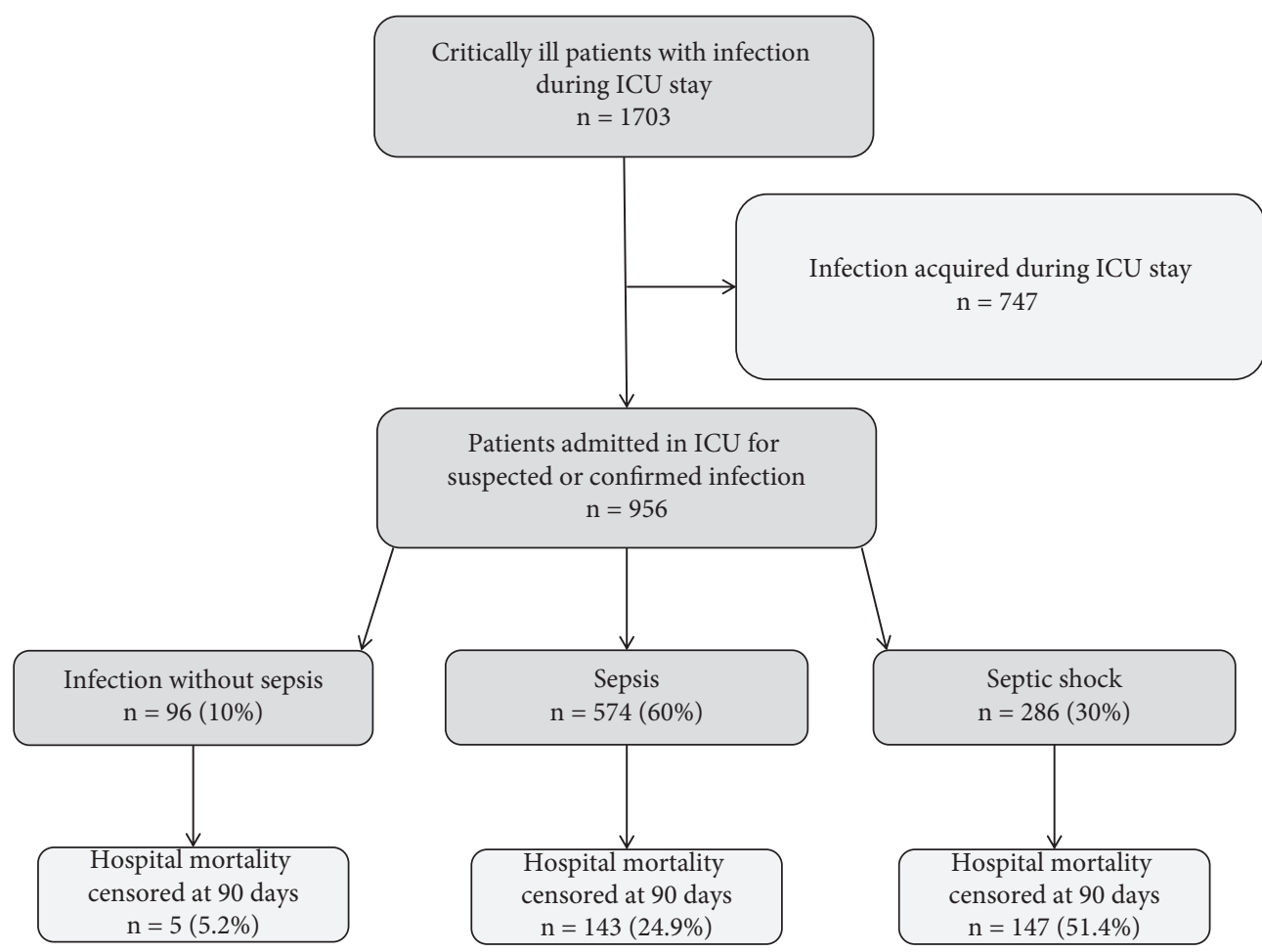

FIGURE 1: Number of patients who were screened and finally included in the analysis. ICU, intensive care unit.

parameter(s) significantly associated with in-hospital mortality within 90 days in SOFA-adjusted analysis (i.e. parameter(s) of interest), we developed a scoring-point system using the regression coefficients of multivariable logistic regression models, dividing the values of the selected parameter(s) into 5 levels and using the Framingham risk development method [32]. To assess this new MACA scoring system, ultimately intended for use in combination with the SOFA score, we estimated the selected parameters' coefficients by reflecting the increase in mortality risk per 1 point of the SOFA score. Parameter intervals were expressed as [a-b), indicating an interval from a to $b$, that is, inclusive of a, but exclusive of $b$. Calibration of this combined "SOFA + MACA" model was assessed by the Hosmer and Lemeshow test [33] and by plotting the observed probability against the predicted probability in deciles. We evaluated the prognostic performance of the combined "SOFA + MACA" model to predict 90 -day mortality by calculating the area under (AUC) the receiver operating characteristic (ROC) curve and using the De Long test to compare with the prognostic performance of a model using only the SOFA score. Statistical testing was performed at the two-tailed $\alpha$ level of 0.05. Data were analyzed using the SAS software package, release 9.4 (SAS Institute, Cary, NC).

\section{Results}

From January $1^{\text {st }} 2015$ to September $30^{\text {th }} 2016,956$ patients were admitted to ICU for infection (see Figure 1). The patients were mostly male $(n=600,62.7 \%)$, and the median age was 63 $[34,35]$ years (see Table 1 ). Within the first 24 hours in ICU, all the patients received both antibiotics and blood volume expansion, 796 patients (83.3\%) received at least $30 \mathrm{~mL} / \mathrm{Kg}$ of vascular filling, 435 (45.5\%) required vasopressors and 413 $(43.2 \%)$ were intubated. Characteristics of the initial infection are detailed in supplemental file 1: infections were mostly community acquired $(n=519,54.3 \%)$ and were pneumoniae for $473(49.5 \%)$ patients, necrotizing dermohypodermitis for 152 (15.9\%), and acute pyelonephritis for 100 (10.5\%). The microbiological sampling was positive in 553 cases (57.8\%); the vast majority of which indicated a bacterial source of infection ( $n=496,51.9 \%)$. Two hundred and ninety-five (30.9\%) patients died within the first 90 days following admission to intensive care.

Missing metabolic parameters were as follows: no patients lacked data for body temperature, 1 (0.1\%) lacked data for glycaemia and 36 (3.8\%) for lactatemia, and 151 (15.8\%) for both cholesterolemia and triglyceridemia. Maximal lactatemia level within 24 hours of admission to ICU was the only metabolic parameter associated with a higher risk of 90-day hospital mortality in both unadjusted (odds ratio (OR), 1.31; 95\%CI, 1.23 to $1.40 ; p<0.001$ ) and SOFA-adjusted (SOFAadjusted OR, 1.17 ; $95 \% \mathrm{CI}, 1.10$ to $1.25 ; p<0.001$; Table 2) analyses. Age and Charlson's comorbidity score were the only two nonmetabolic parameters with an ASD $>25 \%$. They remained significantly associated with 90-day mortality in both unadjusted and SOFA-adjusted analyses (Table 2).

Table 3 presents the MACA model while Figure 2 presents the predicted probabilities of death for each point of the SOFA score and each point of the new model resulting from the sum of the SOFA and MACA scores. Regarding the Hosmer and Lemeshow test, the combined "SOFA + MACA" model exhibited a good calibration (Figure 3, $p=0.47$ ). The 
TABLE 1: Main characteristics of hospital survivors and nonsurvivors to infections at 90 days.

\begin{tabular}{|c|c|c|c|}
\hline & $\begin{array}{c}\text { In-hospital survivors } \\
\text { within } 90 \text { days }(n=661)\end{array}$ & $\begin{array}{c}\text { In-hospital nonsurvivorsg } \\
\text { within } 90 \text { days }(n=295)\end{array}$ & $\operatorname{ASD}(\%)$ \\
\hline Age $\left(\right.$ years) ${ }^{* *}$ & $60(50,70)$ & $66(58,77)$ & 45.6 \\
\hline Male sex, $n(\%)^{* *}$ & $419(63.4)$ & $181(61.4)$ & 4.2 \\
\hline Weight $(\mathrm{kg})^{* *}$ & $76(65 ; 91)$ & $75(65 ; 90)$ & 6.5 \\
\hline \multicolumn{4}{|l|}{ Medical history** } \\
\hline Chronic pulmonary disease & $192(29.0)$ & $88(29.8)$ & 1.7 \\
\hline Diabetes & $149(22.5)$ & $71(24.1)$ & 0.5 \\
\hline Chronic heart failure & $102(15.4)$ & $69(23.4)$ & 20.2 \\
\hline Coronary arterial disease & $86(13.0)$ & $61(20.7)$ & 20.6 \\
\hline Peripheral arterial disease & $64(9.7)$ & $38(12.9)$ & 10.1 \\
\hline Stroke & $90(13.6)$ & $60(20.3)$ & 12.7 \\
\hline Cognitive disorder & $22(3.3)$ & $19(6.4)$ & 14.5 \\
\hline Diffuse connect tissue disease & $38(5.7)$ & $27(9.2)$ & 13.0 \\
\hline Peptic ulcer disease & $11(1.7)$ & $10(3.4)$ & 11.0 \\
\hline Liver disease & $42(6.4)$ & $37(12.5)$ & 21.3 \\
\hline Chronic renal failure (mild to severe) & $46(7.0)$ & $34(11.5)$ & 15.8 \\
\hline Solid cancer & $142(21.5)$ & $86(29.2)$ & 17.7 \\
\hline Leukemia & $48(7.3)$ & $36(12.2)$ & 16.7 \\
\hline Lymphoma & $24(3.6)$ & $23(7.8)$ & 18.0 \\
\hline HIV infection & $1(0.1)$ & $2(0.7)$ & 8.2 \\
\hline Immunocompromised & $135(20.4)$ & $90(30.1)$ & 23.3 \\
\hline Charlson's comorbidity score & $2[1-4]$ & $3[2-6]$ & 60.5 \\
\hline Baseline SOFA & $0[0-0]$ & $0[0-0]$ & 2.9 \\
\hline \multicolumn{4}{|c|}{ Severity scores and metabolic parameters within 24 hours after ICU admission } \\
\hline SOFA $^{* *}$ & $5(3,8)$ & $9(6,13)$ & 96.5 \\
\hline Maximal lactatemia, $(\mathrm{mmol} / \mathrm{L})^{*}$ & $1.6(1.0 ; 2.6)$ & $2.9(1.5 ; 5.1)$ & 69.9 \\
\hline Minimal glycaemia $(\mathrm{g} / \mathrm{L})^{*}$ & $1.1(0.9 ; 1.3)$ & $1.0(0.8 ; 1.3)$ & 15.1 \\
\hline Maximal glycaemia $(\mathrm{g} / \mathrm{L})^{*}$ & $1.6(1.3 ; 2.1)$ & $1.6(1.2 ; 2.2)$ & 4.1 \\
\hline Minimal temperature $\left({ }^{\circ} \mathrm{C}\right)^{*}$ & $36.5(36.0 ; 37.1)$ & $36.4(35.8 ; 37.1)$ & 12.5 \\
\hline Maximal temperature $\left({ }^{\circ} \mathrm{C}\right)^{*}$ & $37.9(37.2 ; 38.6)$ & $37.8(36.8 ; 38.7)$ & 9.8 \\
\hline Total serum cholesterol $(\mathrm{g} / \mathrm{L})^{*}$ & $1.1(0.8 ; 1.4)$ & $0.9(0.7 ; 1.3)$ & 30.3 \\
\hline Total serum triglycerides $(\mathrm{g} / \mathrm{L})^{*}$ & $1.1(0.8 ; 1.7)$ & $1.1(0.8 ; 1.6)$ & 5.5 \\
\hline \multicolumn{4}{|l|}{ Characteristics at hospital discharge $e^{* *}$} \\
\hline ICU LOS, days & $8(4,16)$ & $5(2,14)$ & - \\
\hline Hospital LOS, days & $16(8,29)$ & $7(2,19)$ & - \\
\hline Glasgow outcome scale & $4(4,5)$ & 1 & - \\
\hline Rankin score & $1(0 ; 2)$ & 6 & - \\
\hline
\end{tabular}

Values are expressed as counts (\%) or medians $\left(25^{\text {th }} ; 75^{\text {th }}\right.$ centiles). ASD, absolute standardized difference, ICU, intensive care unit; LOS, length of stay; SOFA : sequential organ failure Assessment. * Missing metabolic parameters were as follows: no patients lacked data for body temperature, $1(0.1 \%)$ lacked data for glycaemia, $36(3.8 \%)$ for lactatemia, and $151(15.8 \%)$ for both cholesterolemia and triglyceridemia. ${ }^{* *}$ No missing data.

TABLE 2: Unadjusted and SOFA-adjusted odds ratio for in-hospital mortality within 90 days of intensive care admission.

\begin{tabular}{|c|c|c|c|c|}
\hline \multirow{2}{*}{ Variable within the first 24 hours } & \multicolumn{2}{|c|}{ Unadjusted analyses } & \multicolumn{2}{|c|}{ SOFA-adjusted analyses } \\
\hline & OR $[95 \% \mathrm{CI}]$ & $p$ value & OR $[95 \% \mathrm{CI}]$ & $p$ value \\
\hline Maximal lactatemia* & $1.31[1.23 ; 1.40]$ & $<0.001$ & $1.17[1.10 ; 1.25]$ & $<0.001$ \\
\hline Minimal glycaemia* & $0.85[0.61 ; 1.17]$ & 0.31 & $0.84[0.60 ; 1.18]$ & 0.32 \\
\hline Maximal glycaemia* & $0.95[0.80 ; 1.13]$ & 0.59 & $0.85[0.70 ; 1.03]$ & 0.089 \\
\hline Minimal temperature* & $0.85[0.75 ; 0.96]$ & 0.009 & $0.95[0.83 ; 1.08]$ & 0.41 \\
\hline Maximal temperature* & $0.91[0.80 ; 1.03]$ & 0.12 & $0.89[0.77 ; 1.01]$ & 0.076 \\
\hline Cholesterol level $^{*}$ & $0.57[0.41 ; 0.80]$ & 0.001 & $0.81[0.59 ; 1.13]$ & 0.21 \\
\hline Triglyceride level* & $1.02[0.89 ; 1.16]$ & 0.83 & $0.90[0.77 ; 1.05]$ & 0.16 \\
\hline Age* $^{*}$ & $1.03[1.02 ; 1.04]$ & $<0.001$ & $1.04[1.03 ; 1.05]$ & $<0.001$ \\
\hline Charlson's comorbidity score* & $1.22[1.16 ; 1.28]$ & $<0.001$ & $1.24[1.17 ; 1.32]$ & $<0.001$ \\
\hline
\end{tabular}

$\mathrm{CI}$, confidence interval; OR: odds ratio; SOFA, sequential organ failure assessment. ${ }^{*}$ Missing parameters were as follows: no patients lacked data for body temperature, age, and Charlson's comorbidity score, $1(0.1 \%)$ lacked data for glycaemia, 36 (3.8\%) for lactatemia, and 151 (15.8\%) for both cholesterolemia and triglyceridemia Bold values highlight the significant $p$ values. 
TABLE 3: The metabolic failure, age, and comorbidity assessment (MACA) score.

\begin{tabular}{|c|c|c|c|c|c|}
\hline \multirow{2}{*}{$\begin{array}{l}\text { Parameters } \\
\text { Maximal lactatemia }(\mathrm{mmol} / \mathrm{L})\end{array}$} & \multicolumn{5}{|c|}{ Categories } \\
\hline & $<1$ & {$[1.0-2.0)$} & {$[2.0-4.0)$} & {$[4.0-6.0)$} & $\geq 6$ \\
\hline Number of patients & 149 & 340 & 256 & 84 & 91 \\
\hline Regression coefficients* & (Ref) & 0.74 & 2.03 & 3.76 & 13.3 \\
\hline Attributed points & 0 & 1 & 2 & 4 & 13 \\
\hline Age & $<40$ & {$[40-55)$} & {$[55-65)$} & {$[65-80)$} & $\geq 80$ \\
\hline Number of patients & 102 & 177 & 227 & 333 & 117 \\
\hline Regression coefficients* & (Ref) & 3.70 & 6.02 & 8.34 & 11.40 \\
\hline Attributed points & 0 & 4 & 6 & 8 & 11 \\
\hline Charlson's comorbidity score & $0-1$ & $2-3$ & $4-5$ & $6-7$ & $\geq 8$ \\
\hline Number of patients & 318 & 337 & 147 & 60 & 94 \\
\hline Regression coefficients* & (Ref) & 2.08 & 4.17 & 6.25 & 10.94 \\
\hline Attributed points & 0 & 2 & 4 & 6 & 11 \\
\hline
\end{tabular}

The metabolic failure, age and comorbidity assessment (MACA) score according to maximal lactatemia within the first 24 hours of intensive care admission and to background characteristics (i.e: age and Charlson's comorbidity score). * Regression coefficients associated with maximal lactatemia, age, or Charlson's score calculated using the Framingham method to reflect the increase associated with one point of the sequential organ failure assessment (SOFA) score. The notation [a-b) indicates an interval from a to $b$, that, is inclusive of a but exclusive of $b$.

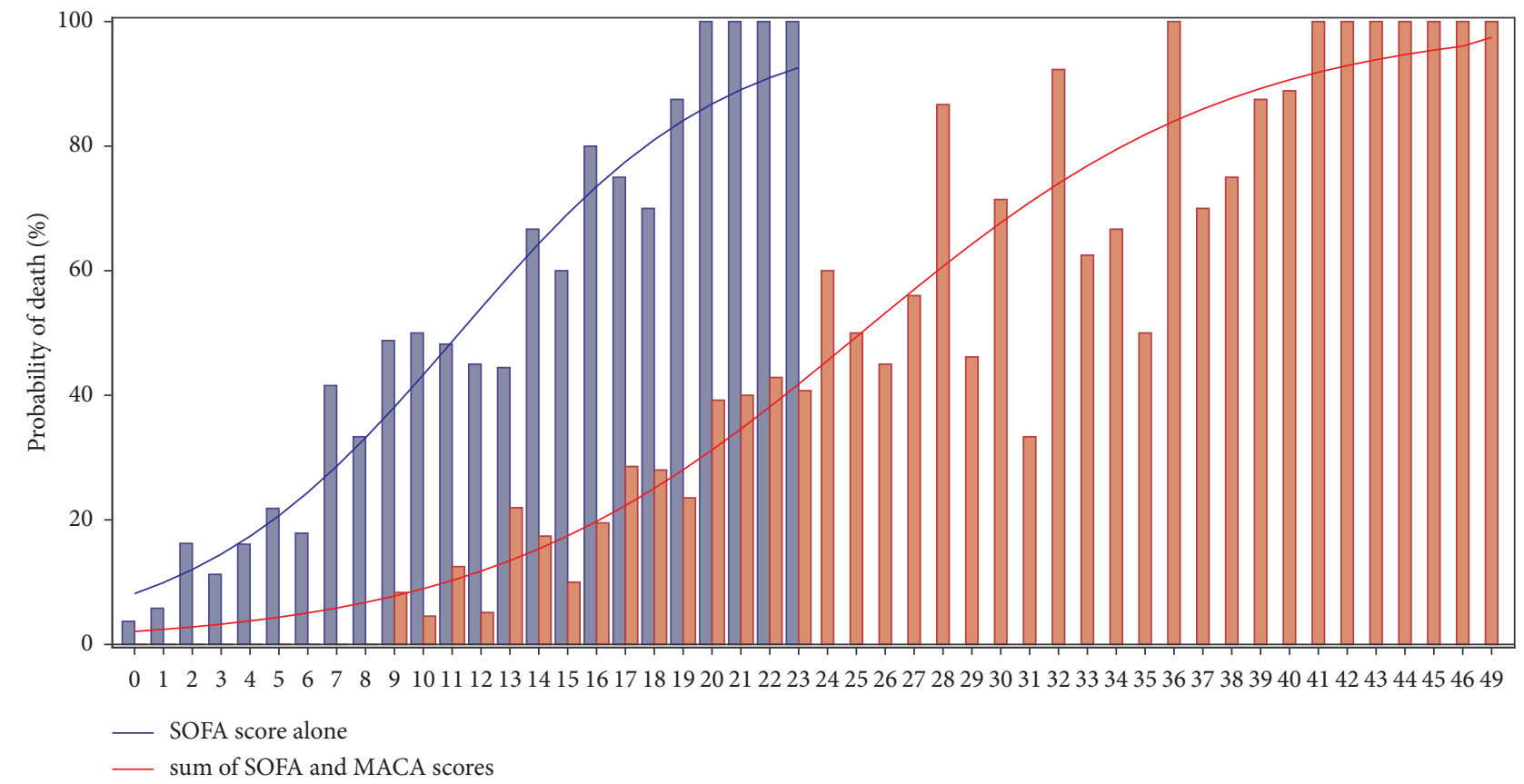

Figure 2: Predicted probability of in-hospital mortality within 90-days of intensive care admission for severity scores. Histograms represents observed probability of death and lines represents predicted probability of death. SOFA, Sequential Organ Failure Assessment; MACA, metabolic failure, age, and comorbidity assessment. No patient had a total SOFA score of 24 points in our study. No patient had a total combined "SOFA + MACA" model score of 50 points or more.

distribution of patients' SOFA score alone and the combined "SOFA + MACA" model according to infection severity is presented in Supplemental file 2.

The performance of the combined "SOFA + MACA" model to predict 90 -day hospital mortality (AUC, $0.81 ; 95 \%$ CI, 0.78 to 0.84 ) was significantly better than the model based solely upon the SOFA score (AUC $0.74 ; 95 \%$ CI, 0.71 to $0.77 ; p<0.001)$.

\section{Discussion}

We report here the first study to assess an integrated measure of sepsis-related metabolic dysfunction integrating seven commonly available clinical and biological markers, age, and the Charlson's comorbidity score (MACA). Among the preselected candidates of metabolic dysfunction, only maximal lactatemia level within the first $24 \mathrm{~h}$ of ICU admission was associated with a higher risk of mortality in the SOFA-adjusted analysis, while the two background characteristics (age and Charlson's comorbidity score) were also associated with a higher risk of mortality in SOFA-adjusted analyses. The performance of a combined "SOFA + MACA" model to predict 90-day hospital mortality was better than the model based solely upon the SOFA score.

The present study is the first to describe a multivariate analysis comparing the relative association between several 


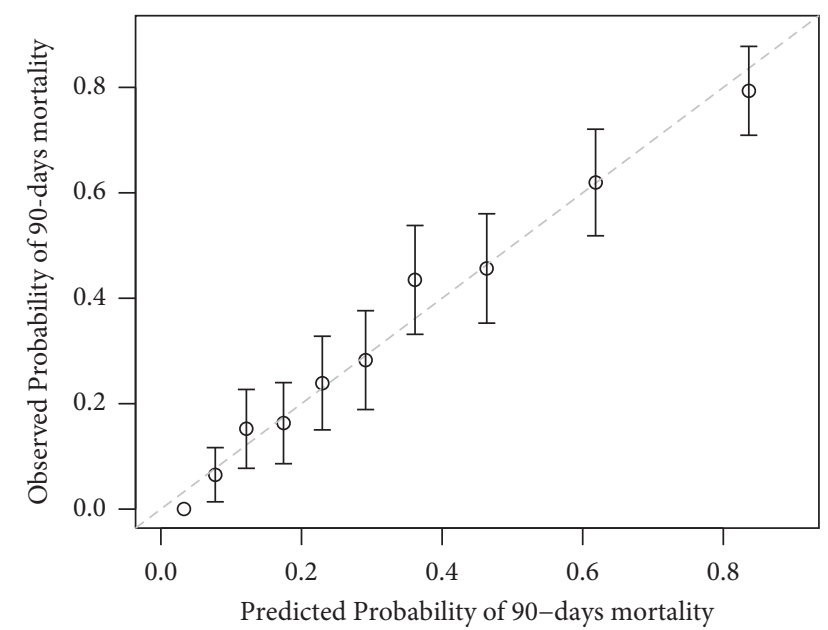

FIgURE 3: Calibration plot of the combined "SOFA + MACA" model to predict short-term mortality. Regarding the Hosmer and Lemeshow test, this combined "SOFA + MACA" model exhibited a good calibration $(p=0.47)$. MACA, metabolic failure, age, and comorbidity assessment; SOFA, sequential organ failure assessment.

metabolic parameters and mortality. Previous studies already showed the prognostic value of hypothermia [13], hypoglycaemia $[14,15]$, hypocholesterolemia [16-18], and hyperlactatemia $[9,10,36-44]$, but none performed a global analysis of their respective impact on mortality. High lactate levels are commonly associated with poor outcome in critically ill patients, including those admitted for sepsis or septic shock $[9,10,36-44]$. Howell et al. have also demonstrated that hyperlactatemia is an independent risk factor of 28-day mortality in a prospective cohort of 1287 patients admitted to the emergency department with an infection [45]. Thus, assessment of plasmatic lactate has already been proposed for risk stratification of patients admitted to the hospital for infection or sepsis $[12,45]$. Some authors have shown the utility of combining lactatemia with the quickSOFA score (qSOFA) in non-ICU patients [46, 47]. Our results further suggest that in combination with the SOFA score, lactatemia measurements may be helpful in deciding which patients could benefit from more proactive treatment strategies and early admission to ICU. First, the present study suggests that patients with isolated lactatemia $\geq 2 \mathrm{mmol} / \mathrm{L}$, or a combination of a lactatemia of [1.0-2.0) $\mathrm{mmol} / \mathrm{L}$ and a total SOFA score of 1 , might be considered as having a cryptic sepsis and should be treated accordingly $[48,49]$. Other studies have also reported that lactatemia levels ostensibly in the "normal range" (i.e. between 1 and $2 \mathrm{mmol} / \mathrm{L}$ ) are associated with short- and long-term mortality in patients with sepsis $[4,50,51]$.

In patients with elevated lactatemia upon admission, previous studies have already shown the advantages of dynamic lactate measurements to predict hospital mortality (namely the "lactime" concept developed by Bakker et al. [52], time-adjusted lactatemia [53], lactate decrease $[34,54,55]$, or lactate normalization [56]), and it has been recommended that normalization of lactatemia may be used as an early therapeutic target in patients with sepsis or septic shock $[31,49,57]$. We nevertheless chose to study the static maximal lactatemia within the first $24 \mathrm{~h}$ of ICU admission, primarily to facilitate its inclusion to a score that could be ultimately combined with the total SOFA score [23].

Our study also found an association between both the plasmatic cholesterol level and the minimal body temperature with short-term mortality in unadjusted analyses. As previously reported, patients with a lower cholesterol level on admission had a higher risk of mortality $[18,58]$. Cholesterol's role in bacterial endotoxin neutralization [59], its impacts on cellular immunity [60], its role in steroid biosynthesis [61], or indeed a combination of these mechanisms may also explain these findings. Moreover, and also as previously published, nonsurvivors were more likely to have a lower temperature on admission [13]. This may be the reflection of the sick euthyroid syndrome observed in the first phase of hormonal impairment [62]. Nonetheless, in contrast to lactatemia, plasmatic cholesterol levels and body temperature were not associated with mortality in SOFAadjusted analyses. The multimodal mechanisms leading to hyperlactatemia in infected patients may be responsible for its particular relevance for short-term outcomes and make it a good candidate to complement the classical assessment of severe infections using the SOFA score. Indeed, rather than a simple reflection of an impaired oxygen delivery leading to an anaerobic metabolism [63,64], lactate production is a sensitive, standalone indicator of a wide range of metabolic impairments at the cellular level. As observed by AlvarezGarcia et al., the link between tissue hypoxia and lactate production in humans with severe infection remains to be demonstrated [65], but lactatemia is essentially the result of an imbalance between lactate production and elimination. On the one hand, the main mechanisms thought to be responsible for an increase in aerobic lactate production during sepsis are an increase in aerobic glycolysis, an inhibition of the pyruvate dehydrogenase, and dysfunction of the mitochondrial electron transport chain [66-68]. On the other hand, the main metabolic alterations proposed as being responsible for reduced lactate extraction from the blood during sepsis are a gluconeogenesis insufficiency in the liver and kidney and a lack of lactate oxidation in the heart and brain [66-68].

It is interesting to note that maximal lactatemia remained significantly associated with a poor prognosis, even if age and the Charlson's score were taken into account. We identified accurate, modifiable (i.e. maximal lactatemia), and nonmodifiable (i.e. age and comorbidities) factors which predict mortality in combination with the SOFA score in patients hospitalized for a restricted list of potentially severe infections (pneumoniae, necrotizing dermohypodermitis, acute pyelonephritis, acute peritonitis, endocarditis, central venous catheter infection, meningitis, and acute osteoarthritis). Recently, other authors have shown the predictive value of age for sepsis-related mortality [69-71], but none demonstrated an equivalence between "modifiable" and "non-modifiable" factors using a scoring-point system of mortality prediction. Thus, it is interesting that in terms of mortality prediction and in this specific population, a lactatemia of $\geq 2 \mathrm{mmol} / \mathrm{L}$ is barely equivalent to an age of 
$\geq 40$ years or a Charlson's comorbidity score of $\geq 2$ (Table 3 ). Moreover, our results showed that patients hospitalized for pneumoniae, necrotizing dermohypodermitis, acute pyelonephritis, acute peritonitis, endocarditis, central venous catheter infection, meningitis, or acute osteoarthritis, with an age $\geq 40$ years or a Charlson's score $\geq 2$, might also be considered as having a cryptic sepsis and should be treated accordingly $[48,49]$.

We are aware of the limitations of our study. First, the single-centre design may limit the external validity of the results. Nevertheless, we observed similar demographic data to previously studied populations [4, 35, 72]. Chronic pulmonary disease, diabetes, and chronic heart and renal failure were the most frequent coexisting disorders [35]. Concerning hospital mortality, we here report a higher mortality rate (31.5\%) than reported by Raith et al. (18.7\%) [72], but one which is similar to that reported in a British cohort [73] or in the ANDROMEDA-SHOCK trial [74]. The severity on admission was similar to other published data, regarding both the SOFA scores $[4,72]$ and the proportion of infection without sepsis or septic shock $[72,73]$. The lower respiratory tract was also the most common infection site $[54,72,73]$, while infection of skin and soft tissues was the second-most common origin of sepsis. This finding could well be due to a local particularity in admissions at the Lille University Hospital, serving as it does as a referral centre for patients with skin and soft tissue infections. Second, our results were obtained from a population of patients admitted to ICU with potentially severe infections (pneumoniae, necrotizing dermohypodermitis, acute pyelonephritis, acute peritonitis, endocarditis, central venous catheter infection, meningitis, and acute osteoarthritis). Thus, they need to be confirmed in further studies before they can be generalized to an unselected population of patients with less-specific suspected or known infection. Third, as it is impossible to determine the precise cause of hyperlactatemia in the context of infection, we did not account for the potential for treatment-related hyperlactatemia in the analyses of our results, such as metformin overdose [75] or $\beta 2$ mimetic administration [76]. Finally, lactatemia measurements were performed with ICU devices as part of local management protocols [77], primarily to avoid mismeasurement due to the time of transport to the laboratory: our results therefore require confirmation with laboratory-based analytical devices for lactatemia measurements but are nevertheless representative of a real-world clinical situation.

\section{Conclusion}

Metabolic dysfunction is a major component of the pathophysiology of severe infections but nevertheless only forms part of the diagnosis of septic shock, not sepsis. Maximal lactatemia within the first 24 hours in ICU, as an adjunct to the SOFA score, is the best predictor of short-term mortality from among seven measurements of metabolic function commonly available in clinical practice. We also developed a new metabolic failure, age, and comorbidity assessment (MACA) score that included this measure of lactatemia, the patient's age, and Charlson's comorbidity score. Used in combination with the SOFA score, this MACA score could help physicians to diagnose cases of cryptic sepsis and may thus be helpful in deciding which patients could benefit from more proactive treatment strategies and early admission to ICU. The accuracy of this new MACA score needs to be evaluated in future studies, but it has the merit of including physiologically relevant, routine measurements to augment an already well-established risk assessment tool.

\section{Abbreviations}

AUC: Area under the curve

BAL: Bronchoalveolar lavage

ICU: Intensive care unit

IQR: Interquartile range

IV: Intravenous

GOS: Glasgow outcome scale

MACA: Metabolic failure, age and comorbidity assessment

OR: Odds ratio

qSOFA: Quick sequential organ failure assessment

ROC: Receiver operating characteristic

SOFA: Sequential organ failure assessment

UCBE: Urine cytobacteriological examination.

\section{Data Availability}

The data are available on reasonable request sent to the corresponding author.

\section{Conflicts of Interest}

The authors declare that they have no conflicts of interest.

\section{Supplementary Materials}

Supplemental file 1: Characteristics of infections leading to intensive care admission among hospital survivors and nonsurvivors. Values are expressed as counts (\%) or medians (25th; 75th centiles). BAL, bronchoalveolar lavage; UCBE, urine cytobacteriological examination. Supplemental file 2. Distribution of the SOFA and MACA scores according to infection severity. SOFA, Sequential Organ Failure Assessment; MACA, Metabolic failure, age and comorbidity assessment. Infection, sepsis and septic shock are defined according to SEPSIS-3 definitions [1]. 1. Singer $M$, Deutschman CS, Seymour CW, Shankar-Hari $M$, Annane D, Bauer $M$, et al. The Third International Consensus definitions for Sepsis and Septic Shock (Sepsis-3). JAMA. 2016; 315:801-10. (Supplementary Materials)

\section{References}

[1] K. Reinhart, R. Daniels, N. Kissoon, F. R. Machado, R. D. Schachter, and S. Finfer, "Recognizing sepsis as a global health priority-a WHO resolution," New England Journal of Medicine, vol. 377, no. 5, pp. 414-417, 2017.

[2] M. Singer, C. S. Deutschman, C. W. Seymour et al., "The third international Consensus definitions for sepsis and septic shock (Sepsis-3)," Journal of the American Medical Association, vol. 315, no. 8, pp. 801-810, 2016. 
[3] C. W. Seymour, V. X. Liu, T. J. Iwashyna et al., "Assessment of clinical criteria for sepsis," Journal of the American Medical Association, vol. 315, no. 8, pp. 762-774, 2016.

[4] M. Shankar-Hari, G. S. Phillips, M. L. Levy et al., "Developing a new definition and assessing new clinical criteria for septic shock," Journal of the American Medical Association, vol. 315, no. 8, pp. 775-787, 2016.

[5] G. H. Van den Berghe, "Acute and prolonged critical illness are two distinct neuroendocrine paradigms," Verhandelingen - Koninklijke Academie voor Geneeskunde van Belgie, vol. 60, no. 6, pp. 487-518, 1998.

[6] J. Pravda, "Metabolic theory of septic shock," World Journal of Critical Care Medicine, vol. 3, no. 2, pp. 45-54, 2014.

[7] S. H. Lee, M. S. Park, B. H. Park et al., "Prognostic implications of serum lipid metabolism over time during sepsis," BioMed Research International, vol. 2015, Article ID 789298, 8 pages, 2015.

[8] M. Shankar-Hari, D. A. Harrison, and K. M. Rowan, "Differences in impact of definitional elements on mortality precludes international comparisons of sepsis epidemiologyA cohort study illustrating the need for standardized reporting," Critical Care Medicine, vol. 44, no. 12, pp. 2223-2230, 2016.

[9] M. E. Mikkelsen, A. N. Miltiades, D. F. Gaieski et al., "Serum lactate is associated with mortality in severe sepsis independent of organ failure and shock," Critical Care Medicine, vol. 37, no. 5, pp. 1670-1677, 2009.

[10] A. D. Nichol, M. Egi, V. Pettila et al., "Relative hyperlactatemia and hospital mortality in critically ill patients: a retrospective multi-centre study," Critical Care, vol. 14, no. 1 , p. R25, 2010.

[11] M. A. Puskarich, B. M. Illich, and A. E. Jones, "Prognosis of emergency department patients with suspected infection and intermediate lactate levels: a systematic review," Journal of Critical Care, vol. 29, no. 3, pp. 334-339, 2014.

[12] B. Casserly, G. S. Phillips, C. Schorr et al., "Lactate measurements in sepsis-induced tissue hypoperfusion," Critical Care Medicine, vol. 43, no. 3, pp. 567-573, 2015.

[13] C. Brun-Buisson, F. Doyon, J. Carlet et al., "Incidence, risk factors, and outcome of severe sepsis and septic shock in adults. A multicenter prospective study in intensive care units. French ICU Group for Severe Sepsis," Journal of the American Medical Association: The Journal of the American Medical Association, vol. 274, no. 12, pp. 968-974, 1995.

[14] NICE-SUGAR Study Investigators, S. Finfer, S. Finfer et al., "Intensive versus conventional glucose control in critically ill patients," New England Journal of Medicine, vol. 360, no. 13, pp. 1283-1297, 2009.

[15] F. Song, L.-J. Zhong, L. Han et al., "Intensive insulin therapy for septic patients: a meta-analysis of randomized controlled trials," BioMed Research International, vol. 2014, Article ID 698265, 10 pages, 2014.

[16] C. Chenaud, P. G. Merlani, P. Roux-Lombard et al., "Low apolipoprotein A-I level at intensive care unit admission and systemic inflammatory response syndrome exacerbation," Critical Care Medicine, vol. 32, no. 3, pp. 632-637, 2004.

[17] M. Wendel, R. Paul, and A. R. Heller, "Lipoproteins in inflammation and sepsis. II. Clinical aspects," Intensive Care Medicine, vol. 33, no. 1, pp. 25-35, 2007.

[18] K. Biller, P. Fae, R. Germann, H. Drexel, A. K. Walli, and P. Fraunberger, "Cholesterol rather than procalcitonin or C-reactive protein predicts mortality in patients with infection," Shock, vol. 42, no. 2, pp. 129-132, 2014.
[19] H. R. Wong, C. J. Lindsell, V. Pettilä et al., “A multibiomarkerbased outcome risk stratification model for adult septic shock," Critical Care Medicine, vol. 42, no. 4, pp. 781-789, 2014.

[20] S. Preau, D. Vodovar, B. Jung et al., "Energetic dysfunction in sepsis: a narrative review," Annals of Intensive Care, vol. 11, no. 1 , p. 104, 2021.

[21] M. M. Levy, M. P. Fink, J. C. Marshall et al., "2001 SCCM/ ESICM/ACCP/ATS/SIS international sepsis definitions conference," Critical Care Medicine, vol. 31, no. 4, pp. 1250-1256, 2003.

[22] M. E. Charlson, P. Pompei, K. L. Ales, and C. R. MacKenzie, "A new method of classifying prognostic comorbidity in longitudinal studies: development and validation," Journal of Chronic Diseases, vol. 40, no. 5, pp. 373-383, 1987.

[23] J.-L. Vincent, R. Moreno, J. Takala et al., "The SOFA (Sepsisrelated Organ Failure Assessment) score to describe organ dysfunction/failure," Intensive Care Medicine, vol. 22, no. 7, pp. 707-710, 1996.

[24] V. Das, P.-Y. Boelle, A. Galbois et al., "Cirrhotic patients in the medical intensive care unit: early prognosis and long-term survival," Critical Care Medicine, vol. 38, no. 11, pp. 2108-2116, 2010.

[25] "Definition and classification of CKD," Kidney International Supplements, vol. 3, no. 1, pp. 19-62, 2013.

[26] R. A. Taplitz, M. L. Ritter, and F. J. Torriani, "Infection prevention and control, and antimicrobial stewardship," in Infectious Diseases, J. Cohen, W. G. Powderly, and S. M. Opal, Eds., pp. 54-61, Elsevier, Amsterdam, Netherlands, Fourth edition, 2017.

[27] T. C. Horan, M. Andrus, and M. A. Dudeck, "CDC/NHSN surveillance definition of health care-associated infection and criteria for specific types of infections in the acute care setting," American Journal of Infection Control, vol. 36, no. 5, pp. 309-332, 2008.

[28] M. Arentz, E. Yim, L. Klaff et al., "Characteristics and Outcomes of 21 Critically Ill Patients with COVID-19 in Washington State," JAMA, vol. 323, 2020.

[29] J. Rankin, "Cerebral vascular accidents in patients over the age of 60: II. Prognosis," Scottish Medical Journal, vol. 2, no. 5, pp. 200-215, 1957.

[30] B. Jennett and M. Bond, "ASSESSMENT OF outcome after severe brain damage A practical scale," The Lancet, vol. 305, no. 7905, pp. 480-484, 1975.

[31] H. B. Nguyen, W. Kuan, M. Batech et al., "Outcome effectiveness of the severe sepsis resuscitation bundle with addition of lactate clearance as a bundle item: a multi-national evaluation," Critical Care, vol. 15, no. 5, p. R229, 2011.

[32] L. M. Sullivan, J. M. Massaro, and R. B. D’Agostino, "Presentation of multivariate data for clinical use: the Framingham Study risk score functions," Statistics in Medicine, vol. 23, no. 10, pp. 1631-1660, 2004.

[33] D. Hosmer and S. Lemeshow, Applied Logistic Regression, N Y Wiley, New York, NY, USA, Second edition, 2000.

[34] J. Chertoff, M. Chisum, L. Simmons, B. King, M. Walker, and J. Lascano, "Prognostic utility of plasma lactate measured between 24 and $48 \mathrm{~h}$ after initiation of early goal-directed therapy in the management of sepsis, severe sepsis, and septic shock," Journal of intensive care, vol. 4, p. 13, 2016.

[35] A. Pavon, C. Binquet, F. Kara et al., "Profile of the risk of death after septic shock in the present era," Critical Care Medicine, vol. 41, no. 11, pp. 2600-2609, 2013. 
[36] A. Javed, F. W. Guirgis, S. A. Sterling et al., "Clinical predictors of early death from sepsis," Journal of Critical Care, vol. 42, pp. 30-34, 2017.

[37] R. Gotmaker, S. L. Peake, A. Forbes, R. Bellomo, and Arise Investigators, "Mortality is greater in septic patients with hyperlactatemia than with refractory hypotension," Shock, vol. 48, no. 3, pp. 294-300, 2017.

[38] T. C. Jansen, J. van Bommel, R. Woodward, P. G. H. Mulder, and J. Bakker, "Association between blood lactate levels, Sequential Organ Failure Assessment subscores, and 28-day mortality during early and late intensive care unit stay: a retrospective observational study," Critical Care Medicine, vol. 37, no. 8, pp. 2369-2374, 2009.

[39] F. A. Husain, M. J. Martin, P. S. Mullenix, S. R. Steele, and D. C. Elliott, "Serum lactate and base deficit as predictors of mortality and morbidity," The American Journal of Surgery, vol. 185 , no. 5, pp. 485-491, 2003.

[40] H. B. Nguyen, E. P. Rivers, B. P. Knoblich et al., "Early lactate clearance is associated with improved outcome in severe sepsis and septic shock," Critical Care Medicine, vol. 32, no. 8, pp. 1637-1642, 2004.

[41] N. I. Shapiro, M. D. Howell, D. Talmor et al., "Serum lactate as a predictor of mortality in emergency department patients with infection," Annals of Emergency Medicine, vol. 45, no. 5, pp. 524-528, 2005.

[42] H. Khosravani, R. Shahpori, H. T. Stelfox, A. W. Kirkpatrick, and K. B. Laupland, "Occurrence and adverse effect on outcome of hyperlactatemia in the critically ill," Critical Care, vol. 13, no. 3, p. R90, 2009.

[43] S. A. Haas, T. Lange, B. Saugel et al., "Severe hyperlactatemia, lactate clearance and mortality in unselected critically ill patients," Intensive Care Medicine, vol. 42, no. 2, pp. 202-210, 2016.

[44] Z. Liu, Z. Meng, Y. Li et al., "Prognostic accuracy of the serum lactate level, the SOFA score and the qSOFA score for mortality among adults with Sepsis," Scandinavian Journal of Trauma, Resuscitation and Emergency Medicine, vol. 27, no. 1, p. 51, 2019.

[45] M. D. Howell, M. Donnino, P. Clardy, D. Talmor, and N. I. Shapiro, "Occult hypoperfusion and mortality in patients with suspected infection," Intensive Care Medicine, vol. 33, no. 11, pp. 1892-1899, 2007.

[46] A. Shetty, S. P. MacDonald, J. M. Williams et al., "Lactate $\geq 2$ $\mathrm{mmol} / \mathrm{L}$ plus qSOFA improves utility over qSOFA alone in emergency department patients presenting with suspected sepsis," Emergency Medicine Australasia, vol. 29, no. 6, pp. 626-634, 2017.

[47] H. Song, H. G. Moon, and S. H. Kim, "Efficacy of quick Sequential Organ Failure Assessment with lactate concentration for predicting mortality in patients with communityacquired pneumonia in the emergency department," Clinical and Experimental Emergency Medicine, vol. 6, no. 1, pp. 1-8, 2019.

[48] A. Rhodes, L. E. Evans, W. Alhazzani et al., "Surviving sepsis campaign: international guidelines for management of sepsis and septic shock," Intensive Care Medicine, vol. 43, no. 3, pp. 304-377, 2016.

[49] M. M. Levy, L. E. Evans, and A. Rhodes, “The surviving sepsis campaign bundle: 2018 update," Intensive Care Medicine, vol. 44, no. 6, pp. 925-928, 2018.

[50] P. Wacharasint, T.-A. Nakada, J. H. Boyd, J. A. Russell, and K. R. Walley, "Normal-range blood lactate concentration in septic shock is prognostic and predictive," Shock, vol. 38, no. 1, pp. 4-10, 2012.
[51] J. Villar, J. H. Short, and G. Lighthall, "Lactate predicts both short- and long-term mortality in patients with and without sepsis," Infectious diseases, vol. 12, 2019.

[52] J. Bakker, P. Gris, M. Coffernils, R. J. Kahn, and J.-L. Vincent, "Serial blood lactate levels can predict the development of multiple organ failure following septic shock," The American Journal of Surgery, vol. 171, no. 2, pp. 221-226, 1996.

[53] A. Nichol, M. Bailey, M. Egi et al., "Dynamic lactate indices as predictors of outcome in critically ill patients," Critical Care, vol. 15, no. 5, p. R242, 2011.

[54] P. Marty, A. Roquilly, F. Vallée et al., "Lactate clearance for death prediction in severe sepsis or septic shock patients during the first 24 hours in Intensive Care Unit: an observational study," Annals of Intensive Care, vol. 3, no. 3, p. 3, 2013.

[55] M. Masyuk, B. Wernly, M. Lichtenauer et al., "Prognostic relevance of serum lactate kinetics in critically ill patients," Intensive Care Medicine, vol. 45, no. 1, pp. 55-61, 2019.

[56] M. A. Puskarich, S. Trzeciak, N. I. Shapiro et al., "Whole blood lactate kinetics in patients undergoing quantitative resuscitation for severe sepsis and septic shock," Chest, vol. 143, no. 6, pp. 1548-1553, 2013.

[57] T. C. Jansen, J. van Bommel, F. J. Schoonderbeek et al., "Early lactate-guided therapy in intensive care unit patients," American Journal of Respiratory and Critical Care Medicine, vol. 182, no. 6, pp. 752-761, 2010.

[58] B. R. Gordon, T. S. Parker, D. M. Levine et al., "Relationship of hypolipidemia to cytokine concentrations and outcomes in critically ill surgical patients," Critical Care Medicine, vol. 29, no. 8, pp. 1563-1568, 2001.

[59] J. F. Berbée, L. M. Havekes, and P. C. Rensen, "Apolipoproteins modulate the inflammatory response to lipopolysaccharide," Journal of Endotoxin Research, vol. 11, no. 2, pp. 97-103, 2005.

[60] M. F. Muldoon, A. Marsland, J. D. Flory, B. S. Rabin, T. L. Whiteside, and S. B. Manuck, "Immune system differences in men with hypo- or hypercholesterolemia," Clinical Immunology and Immunopathology, vol. 84, no. 2, pp. 145-149, 1997.

[61] P. E. Marik, "Critical illness-related corticosteroid insufficiency," Chest, vol. 135, no. 1, pp. 181-193, 2009.

[62] M. Singer, V. De Santis, D. Vitale, and W. Jeffcoate, "Multiorgan failure is an adaptive, endocrine-mediated, metabolic response to overwhelming systemic inflammation," The Lancet, vol. 364, no. 9433, pp. 545-548, 2004.

[63] W. E. Huckabee, "Abnormal resting blood lactate," The American Journal of Medicine, vol. 30, no. 6, pp. 840-848, 1961.

[64] M. H. Weil and A. A. Afifi, "Experimental and clinical studies on lactate and pyruvate as indicators of the severity of acute circulatory failure (shock)," Circulation, vol. 41, no. 6, pp. 989-1001, 1970.

[65] M. Garcia-Alvarez, P. Marik, and R. Bellomo, "Sepsis-associated hyperlactatemia," Critical Care, vol. 18, no. 5, p. 503, 2014.

[66] B. Levy, "Lactate and shock state: the metabolic view," Current Opinion in Critical Care, vol. 12, no. 4, pp. 315-321, 2006.

[67] L. Van Wyngene, J. Vandewalle, and C. Libert, "Reprogramming of basic metabolic pathways in microbial sepsis: therapeutic targets at last?" EMBO Molecular Medicine, vol. 10, no. 8, 2018.

[68] S. Redant, H. Hussein, A. Mugisha et al., "Differentiating hyperlactatemia type A from type B: how does the lactate/ pyruvate ratio help?" Journal of Translational Internal Medicine, vol. 7, no. 2, pp. 43-45, 2019. 
[69] D. Chicco and G. Jurman, "Survival prediction of patients with sepsis from age, sex, and septic episode number alone," Scientific Reports, vol. 10, no. 1, p. 17156, 2020.

[70] A. Dinh, C. Duran, J. Ropers et al., "Pneumonia short treatment (PTC) study group: factors associated with treatment failure in moderately severe community-acquired pneumonia: a secondary analysis of a randomized clinical trial," JAMA Netw Open, vol. 4, no. 10, 2021.

[71] R. R. Bruno, B. Wernly, H. Flaatten et al., "On behalf of the COVIP Study Group: lactate is associated with mortality in very old intensive care patients suffering from COVID-19: results from an international observational study of 2860 patients," Annals of Intensive Care, vol. 11, no. 1, p. 128, 2021.

[72] E. P. Raith, A. A. Udy, M. Bailey et al., "Prognostic accuracy of the SOFA score, SIRS criteria, and qSOFA score for inhospital mortality among adults with suspected infection admitted to the intensive care unit," Journal of the American Medical Association, vol. 317, no. 3, pp. 290-300, 2017.

[73] M. Shankar-Hari, D. A. Harrison, G. D. Rubenfeld, and K. Rowan, "Epidemiology of sepsis and septic shock in critical care units: comparison between sepsis-2 and sepsis-3 populations using a national critical care database," British Journal of Anaesthesia, vol. 119, no. 4, pp. 626-636, 2017.

[74] G. Hernández, G. A. Ospina-Tascón, L. P. Damiani et al., "Effect of a resuscitation strategy targeting peripheral perfusion status vs. serum lactate levels on 28-day mortality among patients with septic shock: the ANDROMEDASHOCK randomized clinical trial," Journal of the American Medical Association, vol. 321, no. 7, pp. 654-664, 2019.

[75] F. Fourrier and A. Seidowsky, "Intoxication par la metformine: mécanismes de toxicité et prise en charge," Reanimation, vol. 19, no. 6, pp. 539-544, 2010.

[76] J. A. Kraut and N. E. Madias, "Lactic acidosis," New England Journal of Medicine, vol. 371, no. 24, pp. 2309-2319, 2014.

[77] O. Noordally and J.-L. Vincent, "Evaluation of a new, rapid lactate analyzer in critical care," Intensive Care Medicine, vol. 25, no. 5, pp. 508-513, 1999. 